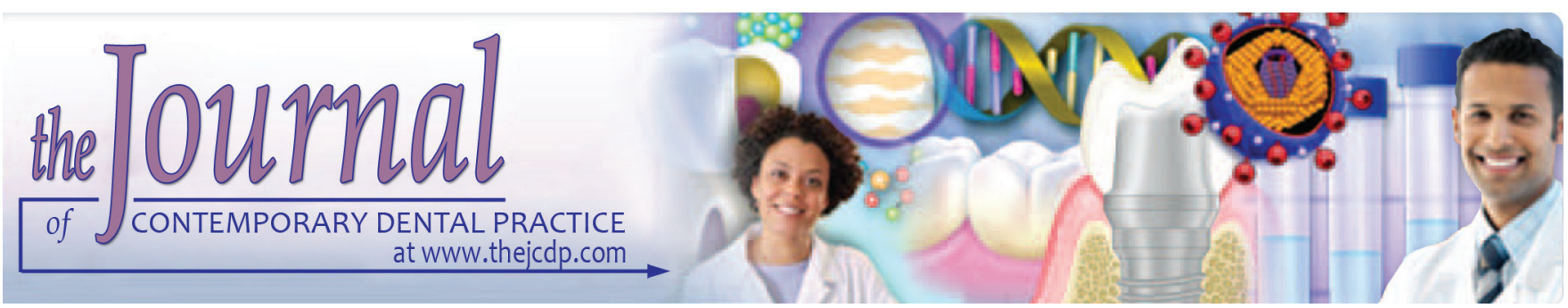

\title{
Comparative Evaluation of Shear Bond Strength and Microleakage of Two Self-adhering Composite Resins: An in vitro Study
}

${ }^{1}$ Priyanka C Panchal, ${ }^{2}$ Karthik Venkataraghavan, ${ }^{3}$ Chirag R Panchal

\begin{abstract}
Introduction: A good dental cement used as repair material should possess chemical binding, easy handling characteristics, minimal marginal breakdown and minimal polymerization shrinkage, high resistance to wear, high cohesive strength, and good color stability. This study was undertaken to compare the in vitro microleakage and shear bond strength among the newly introduced Prime fill flow and Dyad flow.
\end{abstract}

Aim: The aim of this study was to determine the effect on shear bond strength and microleakage of two self-adhesive composite resin.

Materials and methods: Selected specimens were collected and stored in deionized water with an antibactericidal agent, $0.2 \%$ sodium azide, until ready to be used and were randomly divided into two groups: group I: Dyad flow and group II: Prime fill flow; specimens were sheared with a universal testing machine, and the results were calculated in megapascal, specimens were sectioned in a mesiodistal direction at four different locations, and analyzed for leakage (dye penetration) using a stereomicroscope.

Results: The results of the present study showed that acceptable values for Prime fill flow seemed to have least microleakage and high bond strength as compared with Dyad flow.

Conclusion: Here, it can be concluded that there was significant difference in the in vitro microleakage and shear bond strength among the Prime fill flow and Dyad flow.

Clinical significance: Self-etch adhesives when compared with total-etch systems have the advantage of reducing the application time and the number of steps in pediatric dentistry.

\footnotetext{
${ }^{1,2}$ Department of Pedodontics and Preventive Dentistry, College of Dental Sciences \& Research Center, Ahmedabad, Gujarat India

${ }^{3}$ Department Of Orthodontics and Orofacial Orthopedics Squident Kids Dental and Orthodontics, Mumbai, Maharashtra India

Corresponding Author: Priyanka C Panchal, Department of Pedodontics and Preventive Dentistry, College of Dental Sciences \& Research Center, Ahmedabad, Gujarat, India Phone: +917738799881, e-mail: veekart@yahoo.co.in
}

Patients' age and cooperation are not always ideal; the treatment outcome is greatly influenced by the patients' behavior. It is therefore imperative to recede the application time of some materials mostly in pediatric dentistry.

Keywords: Dental cement, Microleakage, Polymerization shrinkage, Resistance, Self-etch adhesive, Shear bond strength.

How to cite this article: Panchal PC, Venkataraghavan K, Panchal CR. Comparative Evaluation of Shear Bond Strength and Microleakage of Two Self-adhering Composite Resins: An In vitro Study. J Contemp Dent Pract 2018;19(9):1082-1086.

Source of support: Nil

Conflict of interest: None

\section{INTRODUCTION}

The vogue for esthetic restorations and substitute materials to replace amalgam restorations has galvanized the pursuit for tooth-colored materials. To accomplish these requirements, resin-bonded composite has been broached as an alternative restorative material. The fruition of these restorations depends on bonding them to hard tooth tissue that will retain the restoration to the cavity preparation and prevent microleakage. ${ }^{1}$ Dentin bonding agents have been revised over the previous 10 years in an endeavor to improve the bonding to the tooth surface and are now available in single-bottle systems to facilitate the ease of use. ${ }^{2}$ Still, in children, it is always difficult to guard the isolation while doing these types of restorations. Recently, new self-etching composite resins have been introduced to the market that manufacturers claim produce good bonding to dentin while being less technique-sensitive that requires no bonding agents. The purpose of this investigation will be:

\section{AIMS AND OBJECTIVES}

- To compare the microleakage and the shear bond strength among the study groups. 
- To scrutinize if there is any relation between microleakage and shear bond strength.

\section{MATERIALS AND METHODS}

\section{Microleakage}

Twenty freshly extracted premolars were collected and reserved in deionized water with an antibactericidal agent, $0.2 \%$ sodium azide, until ready to be used.

Before preparing the teeth for resin placement, the specimen was bathed with a rubber cup and slurry of pumice. Standardized class V cavity preparations were made in the buccal surface at the cementoenamel junction. The separation was made to uniform kidney-shaped outline. The preparation measured $5 \mathrm{~mm}$ long, $3 \mathrm{~mm}$ wide, and $2 \mathrm{~mm}$ deep, which were determined by the entry of bur, with the incisal margin in enamel and the gingival margin in cementum (Fig. 1). The teeth were randomly divided into two groups:

- Group I: Dyad flow

- Group II: Prime fill flow

After fulfillment of the preparations, the bonding agents were applied according to the guidance of the manufacturer. Preparations were filled with self-etching composite in groups I and II. Light curing was done for

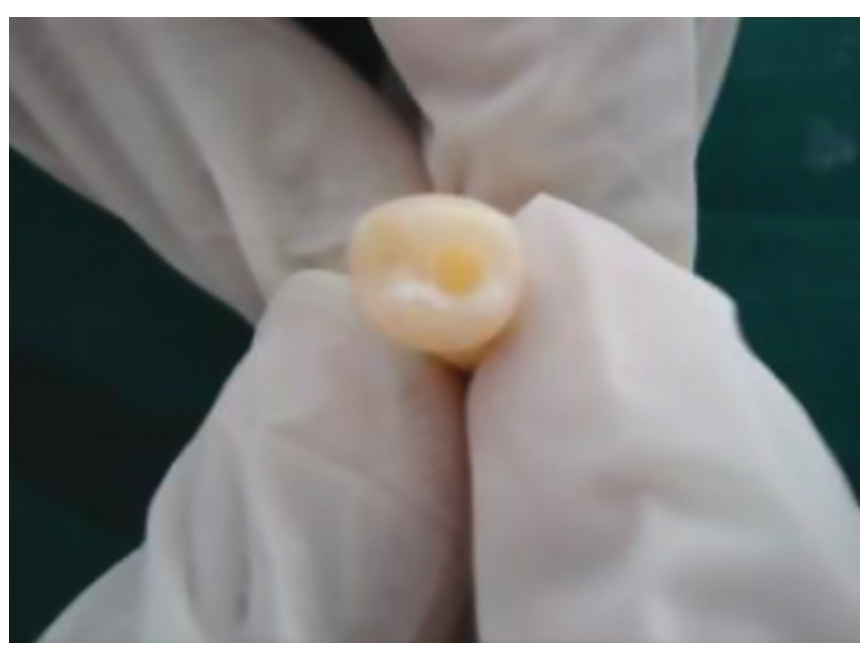

Fig. 1: Prepared tooth with resin
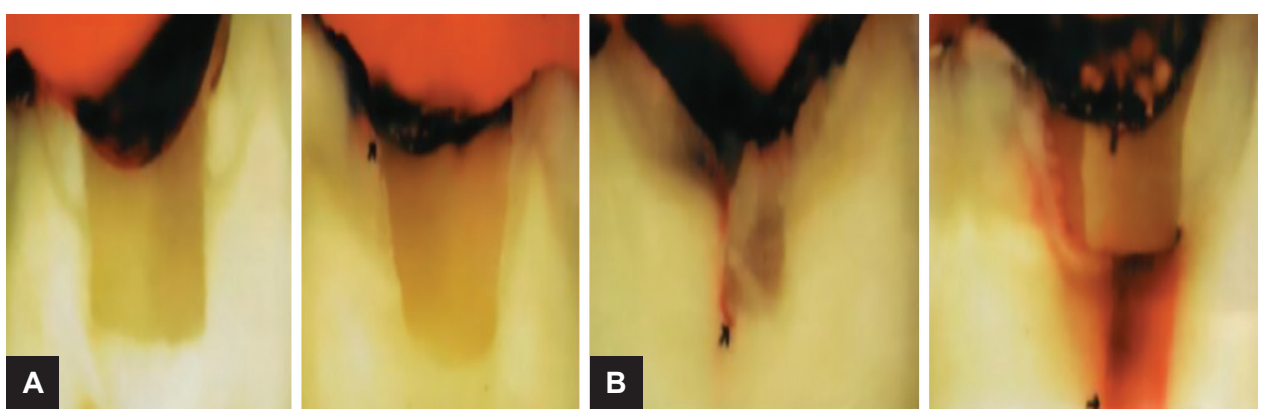

Figs 2A and B: (A) Scores 0 and 1, (B) scores 2 and 3
40 seconds. Then, all specimens were stored in distilled water at $37^{\circ} \mathrm{C}$ for 24 hours.

\section{Microleakage Evaluation}

All groups were arranged for appraisal of microleakage by smearing the complete tooth with one application of nail varnish, except for $1 \mathrm{~mm}$ around the restoration margins. The apices of the teeth were secured with Z100 light-activated composite. Then, all apical areas were covered with a varnish seal. The specimens were then kept in a $\%$ basic Fuchsin dye solution (Fisher Scientific, Fair Lawn, New Jersey, USA) for 24 hours at room temperature, then rinsed with running tap water for 5 minutes. ${ }^{3}$ The specimens were then embedded in a cavity that was made inside cork that was then filled with paraffin wax to hold the specimens and stored in a refrigerator.

Each tooth was sectioned in a mesiodistal direction at four different locations, making a total of 20 specimens for each dentin adhesive system using a slow-speed, watercooled diamond saw. The sections produced were then detached and fixed on a glass slide and the cut surfaces were examined at the incisal and gingival margins under a stereomicroscope. The staining along the tooth restoration interface was based on the degree of dye penetration. The specimens were then gauged based on the graded scoring system with rating values of 0 to 3 . The rating was defined as follows (Fig. 2):

- $0=$ No leakage

- 1 = dye penetration less than half way to the axial wall

- 2 = dye penetration greater than half way to the axial wall

- 3 = dye penetration along axial wall

\section{Shear Strength}

A total of 20 premolar teeth were used in this study. The roots of the teeth were cut and removed. The teeth were stored in de-ionized water until they were ready to be used. The facial surfaces were ground flat to expose dentin with 240- and 400-grit silicon carbide abrasive mounted on a grinding wheel with copious amounts of water. The flattened surfaces of each tooth were 

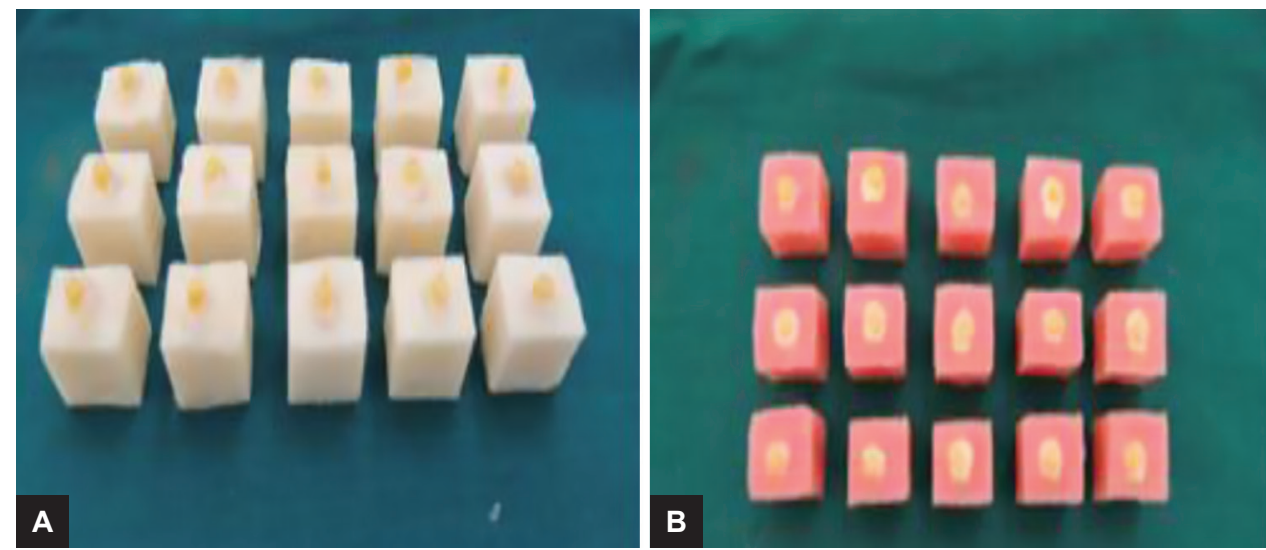

Figs 3 A and B: Mounting of specimens for shear bond strength and composite resin buildup

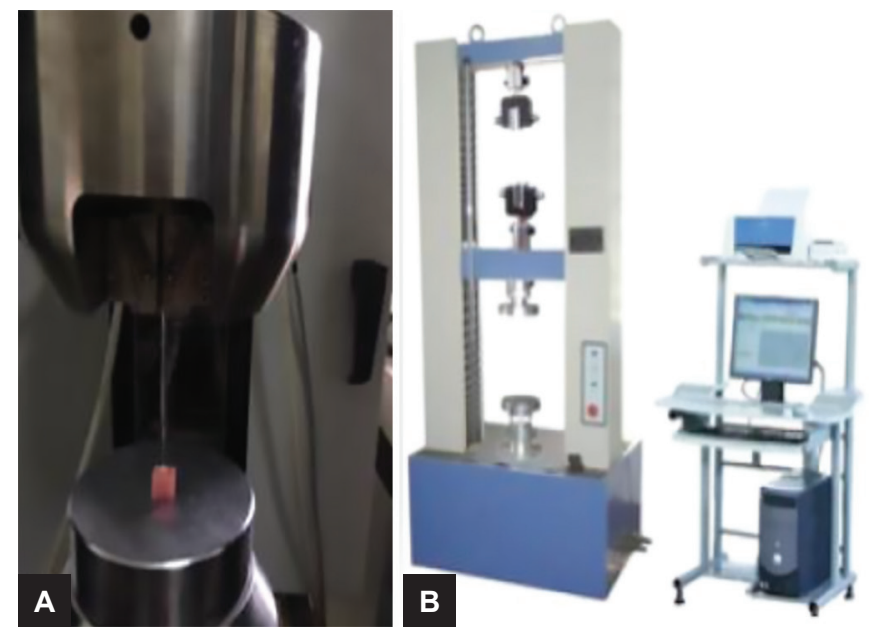

Figs 4A and B: Instron universal testing machine

centered flush horizontally in $35 \mathrm{~mm}$ diameter and 25 $\mathrm{mm}$ depth Teflon molds were held in place with cold cure acrylic resin. Before the bonding procedure, the teeth were again ground slightly using 600-grit silicon carbide abrasive paper to assure clean and fresh surfaces for bonding and to remove any acrylic flashes from the dentin surfaces.

The prepared 20 teeth were divided into two groups. In groups I and II, self-etch composite was applied respectively to the treated dentinal surface through a split Teflon mold, $6 \mathrm{~mm}$ in diameter that approached the surface and extended approximately $3 \mathrm{~mm}$ from the surface, which were firmly attached to the tooth with a metal ring. ${ }^{4}$ Then, the split mold was removed immediately after curing of the restoration (Fig. 3). All specimens were then stored in distilled water for 24 hours at $37^{\circ} \mathrm{C}$.

The shear bond strengths were determined using an Instron universal testing machine at a cross-head speed of $0.5 \mathrm{~mm}$ per minute (Fig. 4). A knife-edge shear probe was attached to the cross-head. Shear forces were documented when the fracture occurred directly from the computer software in $\mathrm{MPa}$.

\section{RESULTS}

A single person carried out all the scoring and microleakage was recorded according to Khera and Chan criteria and shear bond strength test using an Instron universal testing machine at a cross-head speed of $1 \mathrm{~mm}$ per minute.

Table 1 shows the stereomicroscopic dye penetration scores for permanent premolars restored with Prime fill flow and Dyad flow. Based on data available in the table, the following inference could be made:

- The Dyad flow showed the highest microleakage score that ranged between 0 and 3 .

- The Prime fill flow showed the microleakage score that ranged between 0 and 2 .

The data collected were tabulated accordingly and were subjected to statistical analysis using independent sample t-test as shown in Table 2.

Table 1: Microleakage scores in the study groups (scores as per Khera and Chan criteria)

\begin{tabular}{|c|c|c|c|}
\hline \multicolumn{2}{|c|}{ Prime fill flow } & \multicolumn{2}{|c|}{ Dyad flow } \\
\hline Mesial wall & Distal wall & Mesial wall & Distal wall \\
\hline 1 & 0 & 2 & 1 \\
\hline 1 & 1 & 2 & 2 \\
\hline 0 & 2 & 2 & 3 \\
\hline 1 & 0 & 3 & 2 \\
\hline 0 & 0 & 3 & 3 \\
\hline 0 & 1 & 3 & 2 \\
\hline 1 & 1 & 2 & 1 \\
\hline 1 & 2 & 3 & 3 \\
\hline 1 & 0 & 2 & 2 \\
\hline 2 & 2 & 3 & 2 \\
\hline 1 & 0 & 2 & 3 \\
\hline 1 & 2 & 3 & 3 \\
\hline 2 & 2 & 3 & 3 \\
\hline 1 & 1 & 3 & 3 \\
\hline 1 & 0 & 2 & 2 \\
\hline 0 & 0 & 2 & 2 \\
\hline 1 & 1 & 0 & 1 \\
\hline 0 & 0 & 2 & 0 \\
\hline 0 & 1 & 3 & 2 \\
\hline 1 & 1 & 3 & 3 \\
\hline
\end{tabular}


Comparative Evaluation of Shear Bond Strength and Microleakage

Table 2: Independent sample t-test

\begin{tabular}{llllllll}
\hline \multirow{2}{*}{ Group } & Area & $n$ & Mean & $\begin{array}{l}\text { Standard } \\
\text { deviation }\end{array}$ & $\begin{array}{l}\text { Standard } \\
\text { error mean }\end{array}$ & $\begin{array}{l}\text { Mean } \\
\text { difference }\end{array}$ & $p$-value \\
\hline Prime fill flow & Mesial wall & 20 & 0.80 & 0.616 & 0.138 & 0.05 & 0.828 \\
\multirow{3}{*}{ Dyad flow } & Distal wall & 20 & 0.85 & 0.813 & 0.182 & & 0.25 \\
& Mesial wall & 20 & 2.40 & 0.754 & 0.169 & 0.339 \\
\hline
\end{tabular}

Table 3: Shear bond strength test scores in the study groups

\begin{tabular}{ll}
\hline Dyad flow & Prime fill flow \\
\hline 10 & 16 \\
8 & 15 \\
9 & 16 \\
11 & 13 \\
10 & 12 \\
12 & 11 \\
7 & 18 \\
8 & 17 \\
10 & 15 \\
7 & 16 \\
6 & 14 \\
6 & 16 \\
7 & 15 \\
8 & 13 \\
6 & 18 \\
7.5 & 14 \\
6 & 16 \\
7 & 17 \\
7 & 16 \\
8 & 15 \\
\hline
\end{tabular}

The results of intergroup comparison by independent sample t-test revealed that:

- Prime fill flow showed a mean microleakage of 0.80 at mesial wall and 0.85 at distal wall with a mean difference of 0.05 .

- Dyad flow showed a mean microleakage of 2.40 at mesial wall and 2.15 at distal wall with a mean difference of 0.25 .

Therefore, based on obtained mean scores, Prime fill flow seemed to have least microleakage.

Table 3 shows shear bond strength scores for permanent premolars restored with Prime fill flow and Dyad flow. Based on data available in the table, following inference could be made:

- The Dyad flow showed the shear bond strength scores between 6 and $12 \mathrm{kgs}$.
- The Prime fill flow showed the shear bond strength scores between 11 and $18 \mathrm{kgs}$.

The results of intergroup comparison by independent sample t-test revealed that:

- Dyad flow showed a mean shear bond strength of 8.03 kgs with a standard deviation of 1.766 .

- Prime fill flow showed a mean shear bond strength of $15.15 \mathrm{kgs}$ with a standard deviation of 1.872 .

The mean difference sore is 7.13 and p-value of $<0.001$. Therefore, based on obtained mean scores, Prime fill flow seemed to better shear bond strength test (Table 4).

\section{DISCUSSION}

A possible limitation of the experiment is that the results might be influenced by the dye chemical composition (fuchsine) that was used. However, different dyes like fuchsine red, methylene blue, and silver nitrate did not lead to differences in the results obtained by earlier studies. The influence of the dye penetration and its measurement methodology and evaluation criteria on determining microleakage in dentin-restorative interfaces has been obtained by de Camargo et $\mathrm{al}^{5}$ and Browning et al. ${ }^{6}$ First of all, the single-colored agent demonstrates the microleakage without the need for any further introduction of chemical reaction or hazardous radiation. In addition, the researchers can have a range of choices of available dye agents, which allow the method to easily conform to the instruments and methods available at the center in which the research is to be carried out. Often, the evaluation of penetration scores is done on one or more cuts and optical microscope observation. This evaluation method may be less sensitive than three-dimensional evaluation; ${ }^{7}$ however, it is reported that also the use of several (e.g., three) sections of one tooth may avoid underestimation of in vitro microleakage. ${ }^{8}$ Once again, the method is destructive because the specimen is required to be sectioned so that the staining dye layer is measured and recorded under scanning electron microscopy. This neither allows the method to be reproduced nor is the specimen capable

Table 4: Independent sample t-test

\begin{tabular}{lllll}
\hline Group & Sample size & Mean & $\begin{array}{l}\text { Standard } \\
\text { deviation }\end{array}$ & \multicolumn{2}{c}{$\begin{array}{l}\text { Standard error } \\
\text { mean }\end{array}$} & $\begin{array}{l}\text { Mean } \\
\text { difference }\end{array}$ \\
\hline Dyad flow & 20 & 8.03 & 1.766 & 0.395 \\
Prime fill flow & 20 & 15.15 & 1.872 & 0.418 \\
\hline
\end{tabular}


of being long-term assessed. In addition, the results are recorded only from one or two slices obtained from sectioning, which do not represent the whole image of microleakage, which is three-dimensional. Finally, it is highly technique-sensitive and is not able to exclude the diffusion of the dye substance into tooth structures and the restoration from the measurement. The results again do not demonstrate the nature and the patterns of the leakage. ${ }^{9}$

Another criterion of this study was to evaluate and establish a demonstrative value for how strong the bonding of an adhesive system is to dental hard tissues when composites are bonded. Several factors influence in vitro bond strength to dentin, such as the type and age of the teeth, the degree of dentin mineralization, the dentin surface being bonded, the type of bond strength test (shear or tensile), the storage media, and the environmental relative humidity in substrates and testing conditions. These variations could be responsible for the high standard deviation and wide ranges obtained in the present study. ${ }^{3,10,11}$ While, the other finding in this study was that Prime fill flow showed higher bond strength than Dyad flow because of type and amount of solvents, the content and percentage of monomers, and diluents in the mixture influence the bond strength. The filler load or percent mass load differs between products according to manufacturers' technology and is not well described in the adhesives' composition. Nevertheless, there is little information about the shrinkage and stiffness of these filled adhesives after polymerization.

However, this in vitro study needs further in vivo implementation, because this laboratory test was done using extracted teeth without regarding the pulpal pressure and the presence of dentinal fluid under realistic physiological conditions, which may adversely affect dentin bonding. In extracted teeth, the collagen fibrilar network of dentin may collapse and prevent proper resin penetration in dentin. So, long-term clinical studies are required to evaluate the efficacy and durability of these self-etching bonding systems.

\section{CONCLUSION}

Within the limitation of this in vitro study, the following observations were made:

\section{For Microleakage Group}

The results of intergroup comparison by independent sample t-test revealed that:

- Prime fill flow showed a mean microleakage of 0.80 at mesial wall and 0.85 at distal wall with a mean difference of 0.05 .
- Dyad flow showed a mean microleakage of 2.40 at mesial wall and 2.15 at distal wall with a mean difference of 0.25 .

- Therefore, based on obtained mean scores, Prime fill flow seemed to have least microleakage.

\section{For Shear Bond Strength Test Group}

The results of intergroup comparison by independent sample t-test revealed that:

- Prime fill flow showed a mean shear bond strength of $15.15 \mathrm{kgs}$ with a standard deviation of 0.395 .

- Dyad flow showed a mean with a mean shear bond strength of $8.03 \mathrm{kgs}$ with a standard deviation of 1.872 . The mean difference sore is 7.13 and $p$-value of $<0.001$. Therefore, based on obtained mean scores, Prime fill flow seemed to a better shear bond strength test.

Here, it can be concluded that there was a significant difference in the in vitro microleakage and shear bond strength among the Prime fill flow and Dyad flow.

\section{REFERENCES}

1. Miyazaki M, Iwasaki K, Onose H. Adhesion of single application bonding systems to bovine enamel and dentine. Oper Dent 2002 Jan-Feb;27(1):88-94.

2. Kugel G, Ferrari M. The science of bonding: from first to sixth generation. J Am Dent Assoc 2000 Jun;131(Suppl):20S-25S.

3. Cadenaro M, Delise C, Antoniollo F, Navarra OC, Di Lenarda R, Breschi L. Enamel and dentin bond strength following gaseous ozone application. J Adhes Dent 2009 Aug;11(4):287-292.

4. Chan MF, Glyn Jones JC. Significance of thermal cycling in micro leakage analysis for root restorations. J Dent 1994 Oct;22(5):292-295.

5. de Camargo DA, Sinhoreti MA, Correr-Sobrinho L, de Sousa Neto MD, Consani S. Influence of the methodology and evaluation criteria on determining marginal leakage in dentinrestorative interfaces. Clin Oral Investig 2006;10(4):317-323.

6. Browning WD, Brackett WW, Gilpatrick RO. Two-year clinical comparison of a microfilled and a hybrid resin based composite in non-carious class V lesions. Oper Dent 2000 Jan-Feb;25(1):46-50.

7. Gale MS, Darvell BW. Thermal cycling procedures for laboratory testing of dental restorations. J Dent 1999 Feb;27(2):89-99.

8. Raskin A, D'Hoore W, Gonthier S, Degrange M, Dejou J. Reliability of in vitro microleakage test: a literature review. J Adhes Dent 2001 Winter;3(4):295-308.

9. Hilton TJ. Can modern restorative procedures and materials reliably seal cavities? In vitro investigations. Part 1 . Am J Dent 2002 Jun;15(3):198-210.

10. Suryakumari Nujella BP, Choudary MT, Reddy SP, Kiran Kumar M, Gopal T. Comparison of shear bond strength of aesthetic restorative materials. Contemp Clin Dent 2012 Jan-Mar;3(1):22-26.

11. Hilton TJ. Can modern restorative procedures and materials reliably seal cavities? In vitro investigations. Part 2 . Am J Dent 2002 Aug;15(4):279-289. 